\title{
Il cuore senile e lo scompenso cardiaco diastolico dell'anziano
}

\author{
Senile heart and diastolic cardiac insufficiency in the elderly
}

Negli Stati Uniti, il 79\% dei pazienti ricoverati per scompenso cardiaco ha più di 65 anni e un'età media di 75 anni. Tra i pazienti ultraottantacinquenni la percentuale di ricoveri ospedalieri per scompenso cardiaco è aumentata del $25 \%$ dal 1985 al 1995 [1,2] e circa un terzo di tutti i pazienti ricoverati per scompenso cardiaco va incontro a riospedalizzazione nei 90 giorni successivi alla dimissione [3].

In Italia, l'indice di vecchiaia (numero di ultrasessantaquattrenni ogni 100 persone di età inferiore a 15 anni) ha raggiunto il valore del 124,8\%; secondo le previsioni ISTAT, tale indice arriverà al 146,5\% nel 2010 [4]. Negli ultimi vent'anni la popolazione degli ultrasessantacinquenni è passata dal $13,1 \%$ al $18 \%$, mentre la percentuale di ultraottantenni è raddoppiata, giungendo a sfiorare la soglia del $4 \%$ della popolazione totale [4].

Nella popolazione italiana, oltre il $70 \%$ della mortalità complessiva è imputabile alla patologia del sistema cardiocircolatorio [5]. Prevalenza e incidenza di scompenso cardiaco aumentano con l'età: a 65 anni la prevalenza è del 6\%; a 80 anni arriva al 10\%, costituendo la principale causa di ospedalizzazione con una mortalità, nel grande vecchio, del $25 \%$ a un mese e del $50 \%$ a un anno [2].

I principali fattori che interferiscono con il "fisiologico" processo di invecchiamento sono costituiti dalla presenza di patologie cardiache associate e dalle abitudini di vita (attività fisica ridotta e progressiva sedentarietà, alimentazione ipercalorica-iperlipidica con conseguente sovrappeso/ obesità, assunzione di sale, abuso di tabacco ecc.) che agiscono sull'apparato cardiovascolare con effetti diretti e interazioni reciproche $[6,7]$.

È difficile stabilire un confine tra invecchiamento fisiologico del cuore e alterazioni dovute a processi patologici: i due fenomeni sono verosimilmente embricati e si influenzano a vicenda, soprattutto nell'ambito istopatologico e biochimico. Peraltro, una definizione di cuore senile non esiste [8] e la collocazione dell'età senile fra i 70 e gli 85 anni, e dei grandi vecchi oltre gli 85 anni [9], è puramente arbitraria in quanto il dato cronologico può non essere del tutto rispondente alla realtà.

Tuttavia, accettati con riserva questi limiti cronologici, si potrebbe definire l'invecchiamento fisiologico del cuore come quell'insieme di modificazioni anatomo-strutturali che alterano il substrato su cui si innestano meccanismi fisiopatologici specifici, i quali determinano la soglia e la gravità delle malattie cardiovascolari senili [10]. In altre parole, l'invecchiamento cardiaco rende il cuore diverso e differentemente responsivo a tutto ciò che ne può modificare l'equilibrio.

Le principali modificazioni strutturali che rendono ragione di tale diversità si possono riassumere nei seguenti punti:

- rimodellamento vascolare caratterizzato da ispessimento dell'intima e della media che conferisce alla parete arteriosa maggiore rigidità (che dipende, peraltro, anche dall'alterazione endoteliale del tono della muscolatura liscia) con conseguente aumento dalla pressione arteriosa differenziale, della velocità di propagazione dell'onda sfigmica e incremento del postcarico [11];

- incremento complessivo della massa miocardica a causa della perdita di miociti comunque indotta (apoptosi, non apoptosi o necrosi), compensata dall'ipertrofia dei miociti residui, dell'aumento del collagene interstiziale (soprattutto di tipo I) [12], dell'aumento della fibrosi (modificazione dell'elastina che riduce le catene ad alfa-elica) e del tessuto adiposo sottoepicardico;

- aumento dello spessore parietale relativo (rapporto fra lo spessore della parete e il raggio della camera ventricolare), con relativo disequilibrio del microcircolo, e incremento volumetrico degli atri;

- alterazioni del metabolismo energetico con riduzione del numero dei mitocondri, dei livelli intracellulari di adenosina-trifosfato e della creatina-fosfato, minore utilizzo degli acidi grassi, modificazioni dell'omeostasi del calcio e delle proteine coinvolte nell'accoppiamento elettromeccanico [13]; 
- modificazione della fase diastolica per ridotta compliance ventricolare (probabilmente associata ad alterazioni nella ricaptazione del calcio) con progressiva diminuzione del riempimento rapido e incremento di quello tardivo per una compensatoria contrazione atriale più vigorosa;

- modificazione della fase sistolica (mantenuta a riposo da un più vigoroso contributo atriale) soprattutto sotto sforzo per incremento del postcarico, ridotta compliance aortica e stress parietale del ventricolo sinistro [14] con associata diminuzione della regolazione simpatica (ridotta risposta tachicardica allo sforzo).

Complessivamente le modificazioni del cuore senile (tabella 1) indicano che nella maggior parte dei soggetti anziani "sani" il cuore a riposo è adeguato alle necessità emodinamiche e metaboliche dell'organismo; tuttavia, quando si supera la riserva omeostatica, esso è predisposto allo sviluppo di scompenso cardiaco [15] e per quanto detto, poiché l'invecchiamento è associato a una compromissione del riempimento ventricolare sinistro (riduzione del rilasciamento e della compliance), lo scompenso cardiaco diastolico (SCD) costituisce la forma più frequente, tanto da essere presente in più del $50 \%$ dei casi dopo i 75 anni [15] e addirittura nei due terzi delle donne ultraottantenni [16].

Peraltro lo SCD è ancora un'entità in attesa di un più definito inquadramento: in considerazione delle varie cause che lo sottendono (tabella 2 ) è plausibile che esistano varie forme e che sia un'eccessiva semplificazione riunirle in base al dato comune della disfunzione diastolica comunque misurata. Di fatto, la diastole è un processo energiadipendente [17] che per la sua complessità potrebbe essere alterato in una o più fasi, inducendo differenze in grado di influenzare l'entità e la velocità del rilasciamento ventricolare.

L'autonomia nosologica dello SCD può essere individuata in relazione alla modalità di rimodellamento (concentrico), a differenze ultrastrutturali riguardanti le dimensioni dei cardiomiociti, la densità dei miofilamenti e le proteine del citoscheletro, nonché all'espressione di differenti regolatori della matrice collagene [18].

D'altra parte alcuni dati indurrebbero a ritenere che lo SCD sia la fase iniziale di un continuum che porta allo SC a funzione ventricolare depressa: sulla base della riduzione

Tabella 1 Modificazioni funzionali del cuore senile.
Miocardio
- Contrazione sistolica prolungata
- Rilasciamento isovolumetrico prolungato
- Riempimento iniziale ridotto
- Portata cardiaca conservata a riposo
- Sistole atriale prolungata
- Frequenza cardiaca ridotta sotto sforzo
Apparato valvolare
- Insufficienza mitralica (degenerazione mixomatosa)
- Stenosi aortica
Vasi arteriosi
- Aumentata rigidità delle pareti dei grossi vasi
- Riduzione del lume dei piccoli vasi
- Aumento della pressione sistolica
Sistema di conduzione
- Degenerazione delle cellule nodali
- Fibrosi

Tabella 2 Principali cause di scompenso cardiaco diastolico.

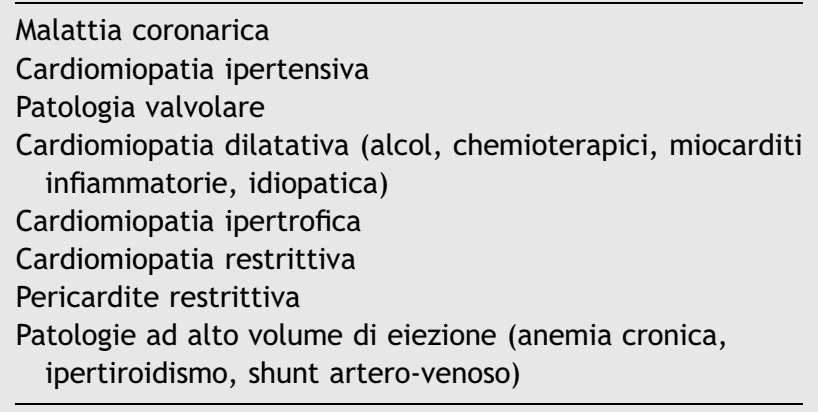

delle velocità di accorciamento delle fibre miocardiche misurate al Doppler tissutale, i soggetti con SCD hanno una funzione ventricolare già compromessa nonostante una frazione di eiezione ventricolare sinistra (LVEF) normale [1923], ed è dimostrata una progressione da forme di ipertrofia concentrica, come nell'ipertensione o nella cardiomiopatia ipertrofica, a forme dilatative.

Indipendentemente da come stiano le cose $[17,24]$ - ed è probabile che entrambe le teorie siano valide - le forme di SCD dovrebbero essere stratificate non solo per il momento patogenetico che le induce, ma anche, e forse soprattutto, in ragione dell'età: è indubbio, infatti, che debbano intendersi come differenti le forme a insorgenza relativamente precoce (per esempio, prima dei 70 anni) e le forme a insorgenza estremamente tardiva (oltre gli $80 \mathrm{anni}$ ) in cui i fattori stessi dell'invecchiamento del cuore, prima ricordati, potrebbero essere chiamati in causa.

Peraltro, se è noto che l'impatto dello SCD aumenta con l'età, a prescindere dal setting, dal tipo di studio (di popolazione, di coorte, di registro ecc.), dalle metodiche d'indagine e dai cut-off usati per definire i criteri di normalità, di converso l'età avanzata rappresenta quasi un prerequisito per questa forma morbosa, al punto che potrebbe sorgere la domanda se lo SCD non sia altro che l'estremo patologico del processo di invecchiamento del cuore, sotteso, quindi, dagli stessi momenti patogenetici.

In altre parole, ci potremmo chiedere se il cuore senile possa essere inteso come l'altra faccia (quella "robusta") delle forme a insorgenza tardiva di SCD, che rappresenterebbero, invece, la faccia "fragile" della stessa medaglia.

Orbene, se andiamo ad analizzare, per esempio, lo SCD conseguente a causa ipertensiva possiamo notare come quelle stesse alterazioni ultrastrutturali che caratterizzano tali forme morbose siano comuni al cuore senile. Al riguardo si fa menzione:

- della manipolazione del calcio citosolico;

- della manipolazione della matrice extracellulare [25];

- della fibrosi sostitutiva ormono-correlata (ruolo del sistema renina-angiotensina-aldosterone, dei peptici natriuretici ecc.);

- del complesso dei sistemi che inducono rimodellamento miocardico [26] e che si traducono nel disordine funzionale della stiffness miocardica ben analizzata dall'ecocardiografia (pattern di alterato rilasciamento ventricolare, pattern di flusso delle vene polmonari, Doppler tissutale, rimodellamento atriale).

Pertanto, mentre per le forme a insorgenza in età relativamente precoce (sotto i 70 anni) devono invocarsi 
meccanismi ben precisi e talora individuabili, per le forme senili si potrebbe ipotizzare che la noxa patogena (perlopiù ipertensione o ischemia silente), interferendo con un substrato già predisposto, non farebbe altro che "estremizzare" i meccanismi di involuzione cardiaca.

In pratica, dunque, il vecchio che presenta dispnea ma senza evidenti segni di congestione e che all'ecocardiogramma ha una LVEF $>50 \%$ e un pattern di flusso transmitralico da alterato rilasciamento $(\mathrm{E} / \mathrm{A}<1)$ ha un cuore "senile" oppure uno SCD?

Una risposta potrebbe venire dal dosaggio dei peptici natriuretici (per una diagnosi certa di SCD oggi si richiedono di fatto - oltre agli altri parametri della "diastologia" più fine derivati per esempio dal Doppler tissutale - il dosaggio del BNP o NT-proBNP) [27], tuttavia nell'anziano e nella donna, guarda caso i soggetti nei quali è più frequente lo $S C D, i$ loro livelli normali sono più elevati [28] e quindi in casi limite il dubbio permane.

Un metodo empirico sarebbe quello ex juvantibus, vale a dire un breve run di diuretico per os: se i sintomi scompaiono in un paio di giorni è probabile che si tratti di scompenso, altrimenti la dispnea deve trovare altra spiegazione (ricordiamo che esiste anche il polmone senile).

In definitiva, però, ci si deve chiedere se sia davvero utile etichettare il "fiatone" del grande vecchio come conseguenza del suo cuore "senile" o di un iniziale SCD o sia, invece, solo curiosità accademica.

\section{Bibliografia}

[1] McCullough PA, Philbin EF, Spertus JA, Kaatz S, Sandberg KR, Weaver WD. Confirmation of a heart failure epidemic: findings from the Resource Utilization Among Congestive Heart Failure (REACH) study. J Am Coll Cardiol 2002;39(1):60-9.

[2] Maclntyre K, Capewell S, Stewart S, Chalmers JW, Boyd J, Finlayson A, et al. Evidence of improving prognosis in heart failure: trends in case fatality in 66547 patients hospitalized between 1986 and 1995. Circulation 2000;102(10):1126-31.

[3] Krumholz HM, Parent EM, Tu N, Vaccarino V, Wang Y, Radford MJ, et al. Readmission after hospitalization for congestive heart failure among Medicare beneficiaries. Arch Intern Med 1997; 157(1):99-104.

[4] ISTAT. Annuario statistico italiano 2001. Roma: ISTAT; 2001.

[5] ISTAT. Condizioni di salute e ricorso ai servizi sanitari. Roma: ISTAT; 2000.

[6] Keller NM, Feit F. Atherosclerotic heart disease in the elderly. Curr Opin Cardiol 1995;10(4):427-33.

[7] Geokas MC, Lakatta EG, Makinodan T, Timiras PS. The aging process. Ann Intern Med 1990;113(6):455-66.

[8] Morpurgo M, Bergamaschini L. Esiste e cos'è il "cuore senile". Ital Heart J Suppl 2004;5(8):623-9.

[9] Di Guglielmo L, Dore R, Raisario A, et al. La diagnostica per immagini dell'invecchiamento cardiaco. Il cuore senile è una realtà? Radiol Med 1999;97:449-60.

[10] Lakatta EG, Levy D. Arterial and cardiac aging: major shareholders in cardiovascular disease enterprises: Part II: the aging heart in health: links to heart disease. Circulation 2003;107(2):346-54.

[11] Goldspink DF, Burniston JG, Tan LB. Cardiomyocyte death and the ageing and failing heart. Exp Physiol 2003;88(3):447-58.
[12] Lakatta EG. Arterial and cardiac aging: major shareholders in cardiovascular disease enterprises: Part III: cellular and molecular clues to heart and arterial aging. Circulation 2003;107(3): $490-7$.

[13] Oxenham $\mathrm{H}$, Sharpe N. Cardiovascular aging and heart failure. Eur J Heart Fail 2003;5(4):427-34.

[14] Kitzman DW. Heart failure with normal systolic function. Clin Geriatr Med 2000;16(3):489-512.

[15] Rich MW. Epidemiology, pathophysiology, and etiology of congestive heart failure in older adults. J Am Geriatr Soc 1997; 45(8):968-74.

[16] Wong WF, Gold S, Fukuyama O, Blanchette PL. Diastolic dysfunction in elderly patients with congestive heart failure. Am J Cardiol 1989;63(20):1526-8.

[17] Zile MR, Brutsaert DL. New concepts in diastolic dysfunction and diastolic heart failure: Part I: diagnosis, prognosis, and measurements of diastolic function. Circulation 2002;105(11):1387-93.

[18] van Heerebeek L, Borbély A, Niessen HW, Bronzwaer JG, van der Velden J, Stienen GJ, et al. Myocardial structure and function differ in systolic and diastolic heart failure. Circulation 2006;113(16):1966-73.

[19] Yu CM, Lin H, Yang H, Kong SL, Zhang Q, Lee SW. Progression of systolic abnormalities in patients with "isolated" diastolic heart failure and diastolic dysfunction. Circulation 2002;105(10): 1195-201.

[20] Yip G, Wang M, Zhang Y, Fung JW, Ho PY, Sanderson JE. Left ventricular long axis function in diastolic heart failure is reduced in both diastole and systole: time for a redefinition? Heart 2002;87(2):121-5.

[21] Nikitin NP, Witte KK, Clark AL, Cleland JG. Color tissue Dopplerderived long-axis left ventricular function in heart failure with preserved global systolic function. Am J Cardiol 2002;90(10): $1174-7$.

[22] Petrie MC, Caruana L, Berry C, McMurray JJ. "Diastolic heart failure" or heart failure caused by subtle left ventricular systolic dysfunction? Heart 2002;87(1):29-31.

[23] Bruch C, Gradaus R, Gunia S, Breithardt G, Wichter T. Doppler tissue analysis of mitral annular velocities: evidence for systolic abnormalities in patients with diastolic heart failure. J Am Soc Echocardiogr 2003;16(10):1031-6.

[24] Aurigemma GP, Zile MR, Gaasch WH. Contractile behavior of the left ventricle in diastolic heart failure: with emphasis on regional systolic function. Circulation 2006;113(2):296-304.

[25] Shirwany A, Weber KT. Extracellular matrix remodeling in hypertensive heart disease. J Am Coll Cardiol 2006;48(1): 97-8.

[26] Díez J, Ertl G. A translational approach to myocardial remodelling. Cardiovasc Res 2009;81(3):409-11.

[27] Paulus WJ, Tschöpe C, Sanderson JE, Rusconi C, Flachskampf FA, Rademakers FE, et al. How to diagnose diastolic heart failure: $a$ consensus statement on the diagnosis of heart failure with normal left ventricular ejection fraction by the Heart Failure and Echocardiography Associations of the European Society of Cardiology. Eur Heart J 2007;28(20):2539-50.

[28] MCDonagh TA, Holmer S, Raymond I, Luchner A, Hildebrant P, Dargie HJ. NT-proBNP and the diagnosis of heart failure: a pooled analysis of three European epidemiological studies. Eur J Heart Fail 2004;6(3):269-73.

Paolo Biagi

Medicina Interna, OORR Valdichiana-Montepulciano, SI E-mail: chipama@libero.it, p.biagi@usl7.toscana.it 